\section{Effect of Rootstocks on Blooming Capacity and Productivity of Apple Cultivars}

\author{
József Racskó $^{1}$ - József Nyéki ${ }^{1}$ - Zoltán Szabó ${ }^{1}$ - \\ Miklós Soltész ${ }^{2}$ - Ervin Farkas ${ }^{3}$ \\ ${ }^{1}$ University of Debrecen, Centre for Agricultural Sciences, \\ Institute for Extension and Development, Debrecen \\ ${ }^{2}$ College of Kecskemét, Faculty of Horticulture, Kecskemét \\ ${ }^{3}$ Apple 2000 Ltd., Nagykutas \\ racsko@helios.date.hu
}

\section{SUMMARY}

The experiment with three different rootstock cultivars was set up in a commercial apple orchard at Nagykutas, which is situated in the western part of Hungary. The aim of our two-year-study was to determine the effect three different growing rootstock cultivars (M9, MM106 and seedling) on the flowering and productivity of 33 apple cultivars. Our observations included the following measurements: the date of the beginning and the end of flowering, flower density, fruit density, fruit numbers per tree and tree productivity. According to our results, it was found that the different growing rootstocks have a great determining effect on the above measurements. Our results showed that the flowering period was similar for all cultivars on the three different rootstocks. However, the flowering and the fruit setting decreased in the order M9, MM106 and seedling rootstocks. In contrast, the fruit number per tree followed, in decreasing order, MM106, seedling and M9 rootstocks.

Keywords: apple, date of flowering, flower density, fruit density, numbers per tree, tree production

\section{INTRODUCTION}

Today, we recognise the importance of the rootstock, which has an essential value for fruit yield (Hrotkó et al., 1995). The rootstock, together with the grafted cultivar, influences the vegetative and generative mass and the profitability of fruit production (Nyéki et al., 1997). Moreover, the rootstock determines the ecological fitness of the tree. Their effects can be recognised in the health status of critical tree phonological stages, tree kilter and tree sensitivity to pests and diseases (Holb, 2000, 2002), moreover in the efficiency of pest and disease management programs and fruit yield (Holb et al., 2003a,b). Rootstocks with good ecological fitness are increasingly important in environmentally-friendly fruit production (Soltész, 1997, 1998).

Several earlier works showed that rootstock has no effect on flowering time of apple cultivars; however, other studies proved that the weaker the growing vigour of a rootstock is, the earlier the start of blooming. The reason is that rootstock with weaker growing vigour has lower heat requirements for blooming compared to that of rootstock with stronger growing vigour (Kozma et al., 2003; Nyéki and Soltész, 1996; Nyéki et al., 2002, 2004; Szabó and Nyéki, 2003).
The aim of our two-year-study was to determine the effect three different growing rootstock cultivars (M9, MM106 and seedling) had on the flowering and productivity of 33 apple cultivars.

\section{MATERIALS AND METHODS}

Assessments were made in a commercial apple orchard at Nagykutas, in the western part of Hungary. The orchard was planted with the tree spacing of $3.2 \times 0.54 \mathrm{~m}$ in 1999 . Trees were grafted on weak, moderate and strong growing vigour of apple rootstocks (M9, MM06 and seedling, respectively). 33 apple cultivars (Granny Smith, Arlett, Green Sliws, Boscoop, Idared, Braeburn Hill Well, Jonagored, Braeburn Scheider, Jonica, Elstar, Mollie's Delicious, Gala del bar, Novayo, Gala Imperial, Pink Lady, Gala Mundial, Prima, Gala Prince, Red Elstar, Gala Royal, Rubinette, Galaxi, Sampion, Gloster, Smoothy, Golden B, Earligold, Golden FGA, Summerred, Golden Reinders, Topáz, Golden Rust, Vista Bella) were grafted on each rootstock, in a total 99 rootstock and grafted cultivar combinations. There were 50 trees for each combination (in total 4950 trees). Trees were pruned to a spindle shape and grass alleyways were used in the rows.

Assessments were made in four replicates with 5 trees per replicate in 2002 and 2003. The assessed parameters were: date of the beginning of bloom, full bloom and end of bloom (petal fall). Moreover, flower and fruit density were observed with a qualitative scale. On the scale, values graded from 110. The scale was created similarly to the study of Holb et al. (2003) for disease severity assessment of apple scab. At harvest time, the number of fruit per tree was also counted for each rootstock and grafted cultivar combination.

\section{RESULTS}

\section{Effects of rootstocks on date of flowering}

Almost all cultivars start to bloom at the same date (Tables 1, 2 and 3, Figures 1-2). In 2003, blooming started and ended 5 and 6 days later, respectively, than these of in 2002. This was due to a long winter and a cold, early spring. However, it can be seen that almost all cultivars grafted on MM106 
and seedling rootstock started to bloom earlier than those grafted on M9 rootstock. Blooming started 1.87 days later on M9 rootstocks compared to the other two. The effect of rootstock on the date of petal fall was stronger than on the date of beginning of bloom (Tables 1, 2 and 3). Petal fall was the earliest on MM106 and the latest on seedling rootstock.

\section{Effects of rootstocks on flower density}

Flower density was the highest on M9 (mean density grade was 6.62) and the lowest on seedling rootstock (mean density grade was 3.62 ). In 2002, the highest flower density value was 5; however, it was 9 in 2003 (Tables 1, 2 and 3). For the average of 33 cultivars, flower density was the highest on M9 and the lowest on seedling rootstock. The difference was large for some cultivars. For instance, flower density of cv. 'Golden Rust' was the highest on MM106 and the lowest on M9 rootstock (Figures 3-4).

\section{Effects of rootstocks on fruit density and numbers per tree}

Fruit density was the highest on M9 and lowest on seedling rootstocks (Tables 1, 2 and 3). However, the number of fruits per tree was the highest on seedling rootstock and the lowest on M9 rootstock. Year difference was considerable on fruit density and number. Yield in 2003 was double that of 2002 (Figures 5-6).

The increasing order of fruit density and number was M9, MM106 and seedling rootstock in most cases in 2002. However, this was changed in 2003, as the highest fruit density was on M9, and the lowest on seedling rootstock (Table 4).

Table 1

Date of beginning of bloom, flower density and the number of fruit per tree on 33 apple cultivars grafted on M9 rootstock (Nagykutas, 2002-2003)

\begin{tabular}{|c|c|c|c|c|c|c|}
\hline \multirow[t]{2}{*}{ Cultivar } & \multicolumn{2}{|c|}{ Date of beginning of bloom } & \multicolumn{2}{|c|}{$\begin{array}{c}\text { Fruit density } \\
(1-10)\end{array}$} & \multicolumn{2}{|c|}{ Number of fruit per tree } \\
\hline & 2002 & 2003 & 2002 & 2003 & 2002 & 2003 \\
\hline Arlet & 02 April & 22 April & 5 & 4 & 41.2 & 51.2 \\
\hline Boskoop & 03 April & 22 April & 4 & 7 & 11.9 & 41.7 \\
\hline Braeburn Hillwell & 09 April & 24 April & 5 & 5 & 21.4 & 15.6 \\
\hline Braeburn Schneider & 15 April & 25 April & 5 & 7 & 13.0 & 10.1 \\
\hline Elstar & 09 April & 24 April & 2 & 9 & 2.2 & 68.4 \\
\hline Gala Delbard & 15 April & 24 April & 3 & 7 & 27.7 & 69.0 \\
\hline Gala Imperial & 15 April & 25 April & 4 & 8 & 8.2 & 54.0 \\
\hline Gala Mundial & 08 April & 24 April & 3 & 9 & 26.5 & 52.2 \\
\hline Gala Prince & 15 April & 24 April & 5 & 9 & 20.9 & 55.1 \\
\hline Gala Royal & 15 April & 25 April & 5 & 7 & 28.0 & 58.0 \\
\hline Galaxy & 09 April & 24 April & 4 & 7 & 11.4 & 44.5 \\
\hline Gloster & 15 April & 23 April & 2 & 5 & 45.4 & 58.4 \\
\hline Golden B & 15 April & 26 April & 4 & 8 & 18.1 & 52.4 \\
\hline Golden FGA & 15 April & 26 April & 4 & 6 & 46.7 & 67.2 \\
\hline Golden Reinders & 09 April & 24 April & 4 & 6 & 38.6 & 38.9 \\
\hline Golden Rust & 05 April & 24 April & 5 & 4 & 33.1 & 37.5 \\
\hline Granny Smith & 15 April & 26 April & 2 & 8 & 15.3 & 63.9 \\
\hline Green Sliws & 03 April & 23 April & 5 & 4 & 16.9 & 32.4 \\
\hline Idared & 02 April & 22 April & 4 & 8 & 7.0 & 43.9 \\
\hline Jonagored & 08 April & 24 April & 4 & 8 & 29.3 & 51.4 \\
\hline Jonica & 03 April & 23 April & 5 & 8 & 15.0 & 48.5 \\
\hline Mollie's Delicious & 09 April & 23 April & 4 & 7 & 10.1 & 43.6 \\
\hline Novayo & 03 April & 23 April & 4 & 7 & 9.3 & 47.1 \\
\hline Pink Lady & 05 April & 23 April & 5 & 6 & 74.5 & 50.7 \\
\hline Prima & 02 April & 22 April & 5 & 5 & 55.8 & 54.1 \\
\hline Red Elstar & 15 April & 24 April & 2 & 7 & 21.1 & 72.6 \\
\hline Rubinette & 08 April & 23 April & 5 & 6 & 51.0 & 33.2 \\
\hline Smoothee & 09 April & 25 April & 3 & 9 & 44.3 & 63.7 \\
\hline Snygold (Earligold) & 03 April & 23 April & 5 & 4 & 20.3 & 29.4 \\
\hline Summerred & 08 April & 23 April & 5 & 6 & 65.4 & 35.3 \\
\hline Šampion & 08 April & 23 April & 5 & 6 & 33.6 & 38.3 \\
\hline Topaz & 08 April & 23 April & 5 & 4 & 23.0 & 52.5 \\
\hline Vista Bella & 05 April & 22 April & 1 & 8 & 6.1 & 48.2 \\
\hline
\end{tabular}


Table 2

Date of beginning of bloom, flower density and the number of fruit per tree on 33 apple cultivars grafted on MM106 rootstock

(Nagykutas, 2002-2003)

\begin{tabular}{|c|c|c|c|c|c|c|}
\hline \multirow[t]{2}{*}{ Cultivar } & \multicolumn{2}{|c|}{ Date of beginning of bloom } & \multicolumn{2}{|c|}{$\begin{array}{c}\text { Fruit density } \\
(1-10) \\
\end{array}$} & \multicolumn{2}{|c|}{ Number of fruit per tree } \\
\hline & 2002 & 2003 & 2002 & 2003 & 2002 & 2003 \\
\hline Arlet & 03 April & 22 April & 5 & 5 & 29.0 & 66.6 \\
\hline Boskoop & 05 April & 23 April & 2 & 3 & 2.1 & 10.6 \\
\hline Braeburn Hillwell & 09 April & 24 April & 4 & 3 & 72.4 & 13.3 \\
\hline Braeburn Schneider & 09 April & 24 April & 5 & 3 & 69.6 & 6.5 \\
\hline Elstar & 09 April & 24 April & 1 & 7 & 2.2 & 80.0 \\
\hline Gala Delbard & 08 April & 23 April & 2 & 9 & 30.3 & 67.3 \\
\hline Gala Imperial & 15 April & 24 April & 3 & 9 & 20.4 & 72.7 \\
\hline Gala Mundial & 08 April & 24 April & 4 & 7 & 46.8 & 83.7 \\
\hline Gala Prince & 09 April & 23 April & 2 & 9 & 17.8 & 75.0 \\
\hline Gala Royal & 09 April & 24 April & 1 & 9 & 17.0 & 84.0 \\
\hline Galaxy & 09 April & 24 April & 3 & 6 & 28.1 & 75.0 \\
\hline Gloster & 15 April & 24 April & 1 & 5 & 19.9 & 54.2 \\
\hline Golden B & 08 April & 24 April & 4 & 6 & 24.3 & 44.9 \\
\hline Golden FGA & 08 April & 25 April & 2 & 5 & 73.8 & 44.1 \\
\hline Golden Reinders & 09 April & 24 April & 3 & 6 & 60.0 & 54.4 \\
\hline Golden Rust & 08 April & 24 April & 2 & 9 & 30.4 & 85.0 \\
\hline Granny Smith & 15 April & 26 April & 1 & 7 & 10.4 & 46.4 \\
\hline Green Sliws & 02 April & 22 April & 5 & 3 & 24.6 & 45.1 \\
\hline Idared & 02 April & 22 April & 4 & 8 & 12.9 & 51.0 \\
\hline Jonagored & 05 April & 23 April & 4 & 6 & 20.4 & 45.8 \\
\hline Jonica & 05 April & 22 April & 3 & 8 & 18.9 & 62.1 \\
\hline Mollie's Delicious & 05 April & 22 April & 5 & 2 & 23.5 & 29.8 \\
\hline Novayo & 05 April & 22 April & 4 & 7 & 20.2 & 47.0 \\
\hline Pink Lady & 05 April & 23 April & 3 & 7 & 34.6 & 51.3 \\
\hline Prima & 02 April & 22 April & 4 & 4 & 60.0 & 60.8 \\
\hline Red Elstar & 09 April & 23 April & 1 & 7 & 2.1 & 74.8 \\
\hline Rubinette & 05 April & 22 April & 4 & 5 & 97.3 & 54.5 \\
\hline Smoothee & 08 April & 24 April & 3 & 6 & 45.4 & 54.1 \\
\hline Snygold (Earligold) & 02 April & 22 April & 3 & 3 & 21.3 & 36.6 \\
\hline Summerred & 02 April & 22 April & 5 & 2 & 84.7 & 7.8 \\
\hline Šampion & 03 April & 22 April & 5 & 5 & 44.0 & 41.7 \\
\hline Topaz & 05 April & 22 April & 3 & 6 & 20.1 & 48.8 \\
\hline Vista Bella & 05 April & 23 April & 2 & 7 & 10.4 & 51.9 \\
\hline
\end{tabular}


Table 3

Date of beginning of bloom, flower density and the number of fruit per tree on 33 apple cultivars grafted on seedling rootstock (Nagykutas, 2002-2003)

\begin{tabular}{|c|c|c|c|c|c|c|}
\hline \multirow[t]{2}{*}{ Cultivar } & \multicolumn{2}{|c|}{ Date of beginning of bloom } & \multicolumn{2}{|c|}{$\begin{array}{c}\text { Fruit density } \\
\text { (1-10) }\end{array}$} & \multicolumn{2}{|c|}{ Number of fruit per tree } \\
\hline & 2002 & 2003 & 2002 & 2003 & 2002 & 2003 \\
\hline Arlet & 03 April & 22 April & 4 & 3 & 34.2 & 30.4 \\
\hline Boskoop & 03 April & 22 April & 3 & 1 & 4.4 & 1.3 \\
\hline Braeburn Hillwell & 09 April & 24 April & 3 & 3 & 32.9 & 29.7 \\
\hline Braeburn Schneider & 09 April & 24 April & 5 & 5 & 30.1 & 36.5 \\
\hline Elstar & 09 April & 24 April & 2 & 1 & 14.0 & 39.9 \\
\hline Gala Delbard & 08 April & 23 April & 4 & 6 & 19.2 & 58.4 \\
\hline Gala Imperial & 15 April & 24 April & 3 & 6 & 44.7 & 72.5 \\
\hline Gala Mundial & 08 April & 24 April & 3 & 7 & 27.8 & 58.1 \\
\hline Gala Prince & 09 April & 24 April & 4 & 6 & 90.0 & 53.5 \\
\hline Gala Royal & 08 April & 23 April & 4 & 6 & 76.1 & 81.6 \\
\hline Galaxy & 08 April & 23 April & 2 & 4 & 36.4 & 50.1 \\
\hline Gloster & 15 April & 24 April & 1 & 3 & 13.5 & 28.6 \\
\hline Golden B & 09 April & 25 April & 3 & 3 & 86.9 & 41.5 \\
\hline Golden FGA & 08 April & 25 April & 3 & 2 & 29.7 & 24.3 \\
\hline Golden Reinders & 09 April & 24 April & 3 & 3 & 26.3 & 66.9 \\
\hline Golden Rust & 05April & 24 April & 3 & 6 & 30.3 & 72.4 \\
\hline Granny Smith & 08 April & 24 April & 2 & 4 & 32.0 & 16.6 \\
\hline Green Sliws & 03 April & 23 April & 5 & 2 & 31.6 & 81.6 \\
\hline Idared & 02 April & 22 April & 4 & 4 & 70.4 & 32.1 \\
\hline Jonagored & 03 April & 23 April & 3 & 3 & 17.9 & 45.7 \\
\hline Jonica & 05 April & 22 April & 4 & 2 & 30.3 & 39.4 \\
\hline Mollie's Delicious & 08 April & 23 April & 3 & 2 & 35.1 & 29.6 \\
\hline Novayo & 03 April & 22 April & 4 & 3 & 25.8 & 42.6 \\
\hline Pink Lady & 05 April & 23 April & 3 & 5 & 28.2 & 73.0 \\
\hline Prima & 03 April & 23 April & 3 & 5 & 19.9 & 71.6 \\
\hline Red Elstar & 09 April & 23 April & 1 & 2 & 25.0 & 41.9 \\
\hline Rubinette & 05 April & 23 April & 3 & 1 & 103.0 & 28.1 \\
\hline Smoothee & 15 April & 26 April & 2 & 4 & 30.2 & 55.4 \\
\hline Snygold (Earligold) & 03 April & 23 April & 4 & 2 & 23.7 & 46.9 \\
\hline Summerred & 03 April & 23 April & 2 & 5 & 5.6 & 39.1 \\
\hline Šampion & 03 April & 22 April & 5 & 5 & 35.4 & 76.8 \\
\hline Topaz & 08 April & 23 April & 3 & 3 & 10.1 & 39.7 \\
\hline Vista Bella & 05 April & 22 April & 2 & 4 & 15.3 & 44.7 \\
\hline
\end{tabular}


Figure 1: Distribution of apple cultivars according to marginality of the beginning of bloom on M9 rootstock (MM106 rootstock)

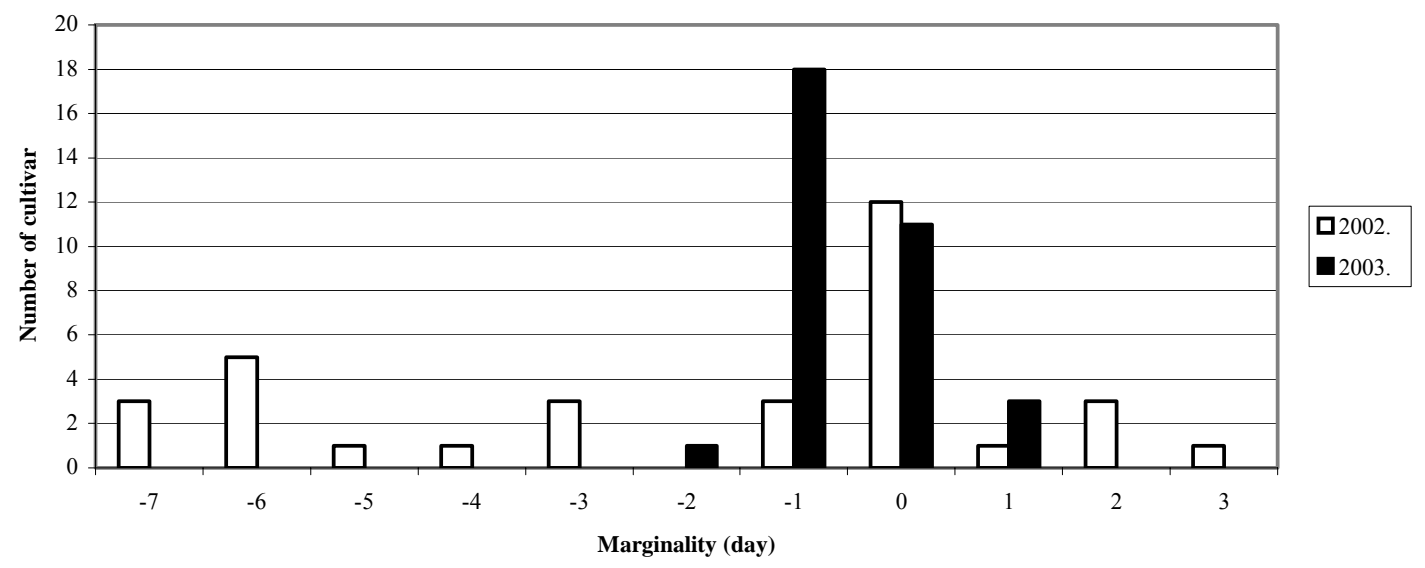

Figure 2: Distribution of apple cultivars according to marginality of the beginning of bloom on M9 rootstock (seedling rootstock)

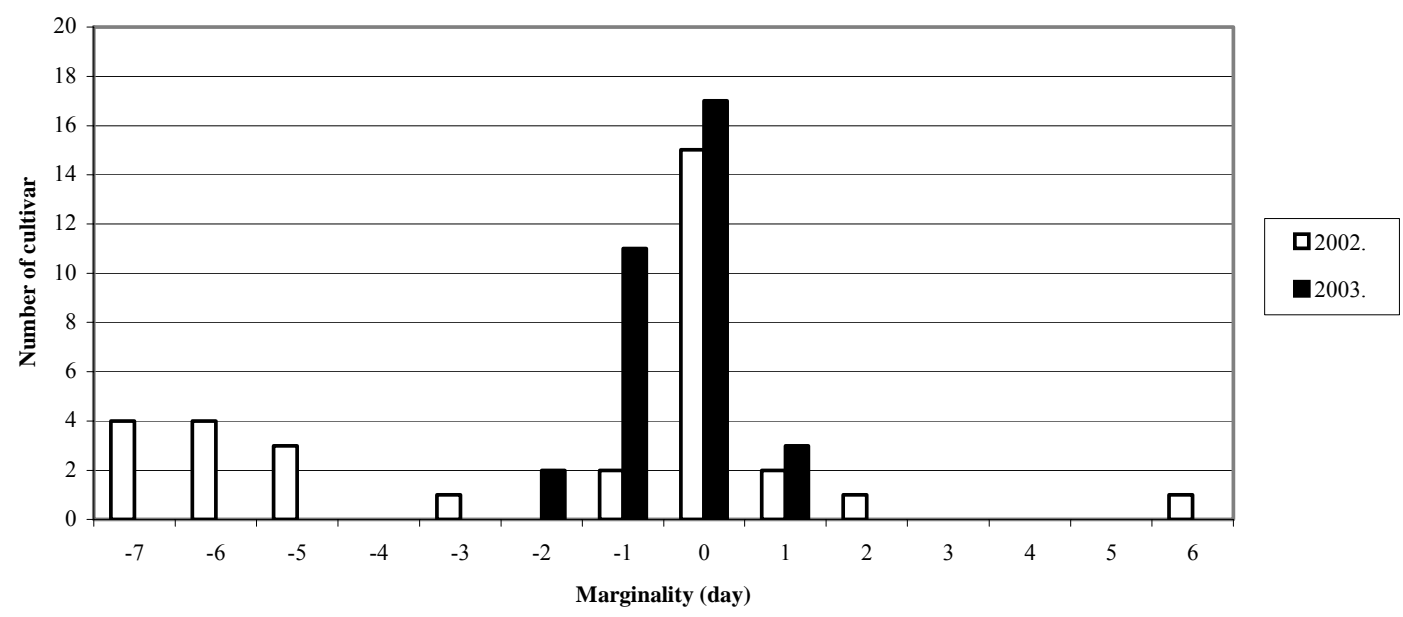

Figure 3: Classification of cultivars according to flower density (2002)

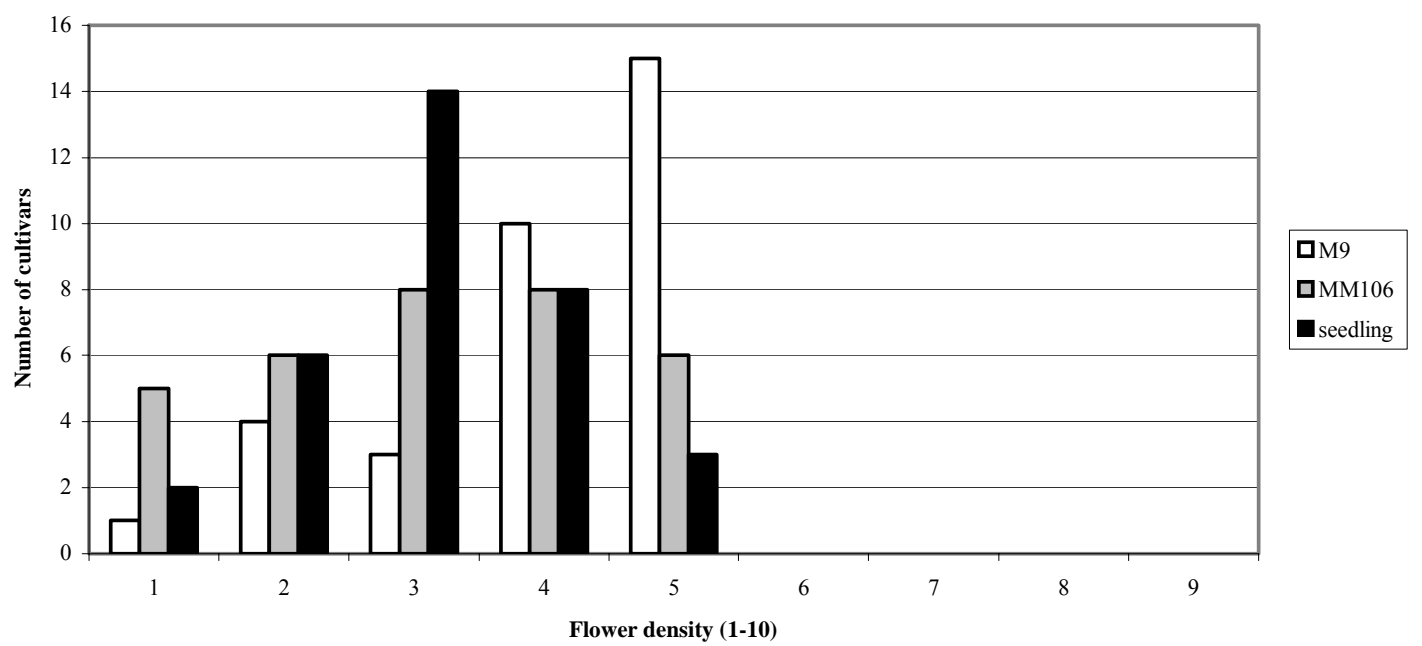


Figure 4: Classification of cultivars according to flower density (2003)

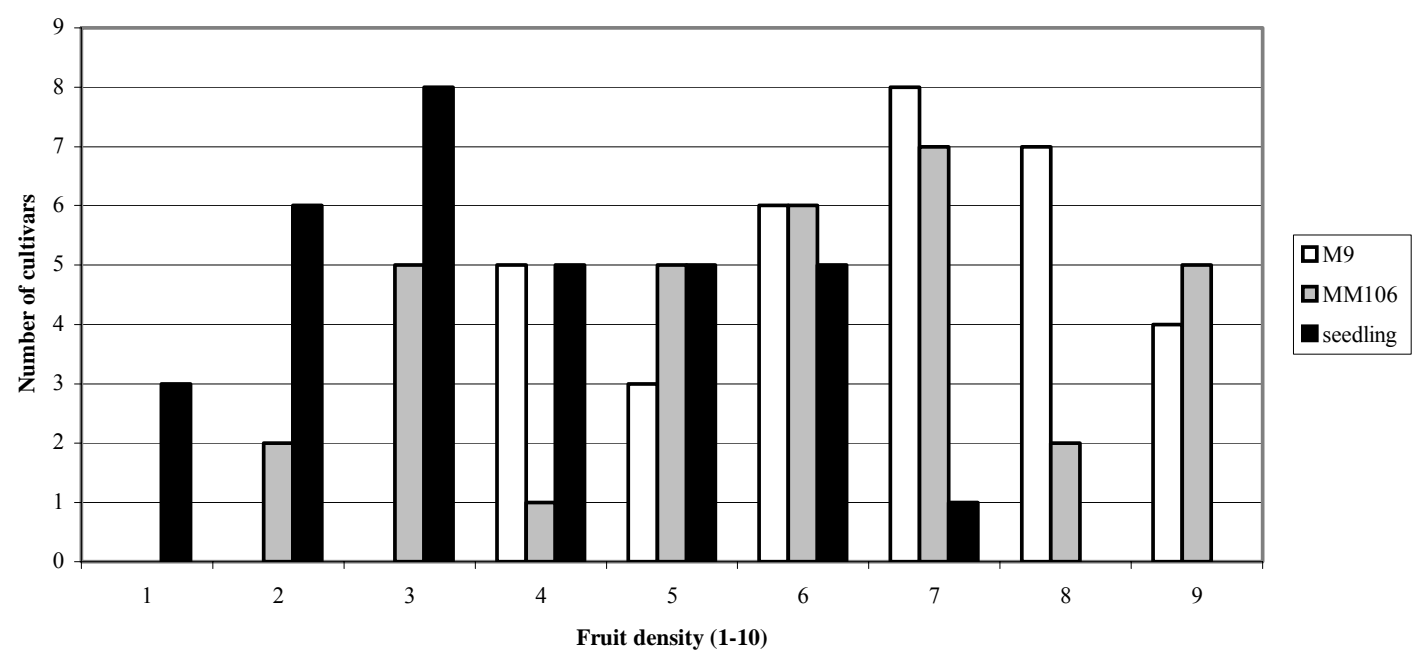

Figure 5: Classification of cultivars according to fruit production per tree (2002)

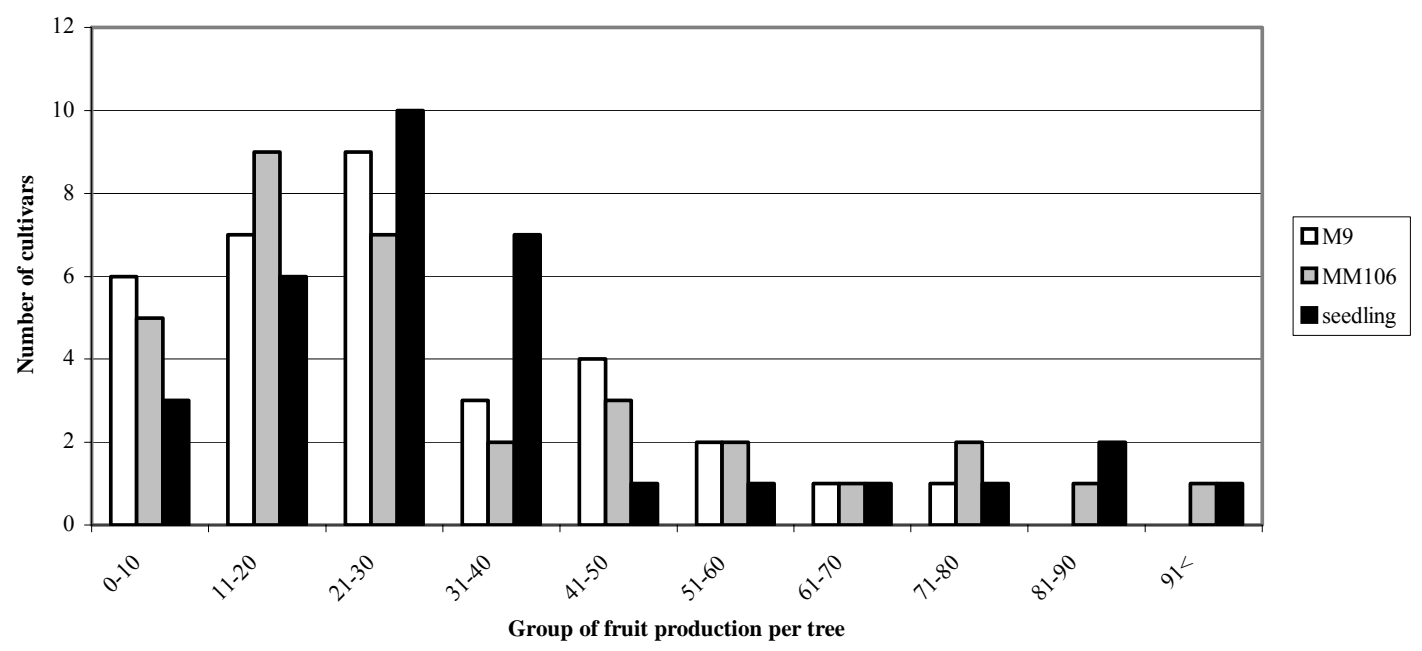

Figure 6: Classification of cultivars according to fruit production per tree (2003)

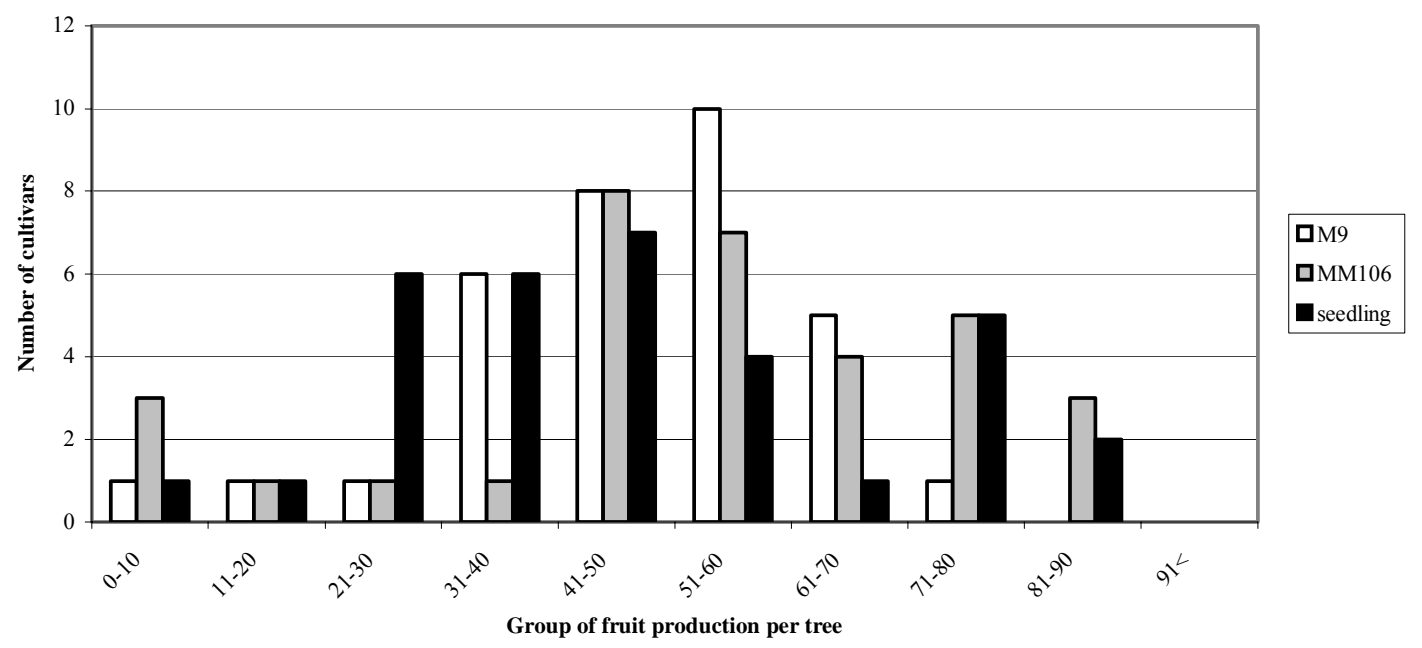


Effect of rootstock on apple tree production

(Nagykutas, Hungary, 2002-2003)

\begin{tabular}{|c|c|c|c|c|}
\hline \multirow{2}{*}{ Order } & \multicolumn{2}{|c|}{2002} & \multicolumn{2}{|c|}{2003} \\
\hline & Number of cultivar & Percent of cultivar & Number of cultivar & Percent of cultivar \\
\hline M9<MM106<seedling & 10 & 31 & 6 & 19 \\
\hline seedling $<$ M9 $<$ MM106 & 7 & 21 & 8 & 24 \\
\hline MM106<M9<seedling & 4 & 12 & 3 & 9 \\
\hline seedling $<$ MM106 $<$ M9 & 4 & 12 & 8 & 24 \\
\hline M9<seedling $<$ MM106 & 3 & 9 & 5 & 15 \\
\hline MM106 $<$ seedling $<$ M9 & 2 & 6 & 1 & 3 \\
\hline M9=MM106<seedling & 1 & 3 & 0 & 0 \\
\hline M9=seedling $<$ MM106 & 1 & 3 & 0 & 0 \\
\hline MM106=seedling $<$ M9 & 1 & 3 & 2 & 6 \\
\hline Total & 33 & 100 & 33 & 100 \\
\hline
\end{tabular}

\section{CONCLUSIONS}

Almost all cultivars bloomed at the same date; however, there were large yearly differences. Rootstock seems to have a considerable effect on the date of petal fall and it was much later on dwarf rootstock than on the other two. Flower and fruit densities are higher on dwarf rootstock than on semidwarf or seedling rootstocks. However, fruit yield per tree is lower on dwarf rootstock than on the other ones.

\section{REFERENCES}

Holb, I. (2002): Epidemiological characteristics of the disease. 2955. In: Holb, I. (eds.), Apple scab: biology, forecasting and control. Szaktudás Kiadó Ház Press, Budapest

Holb, I. J. (2000): Disease progress of apple scab caused by Venturia inaequalis in environmentally friendly growing systems. Int. J. of Hort. Science, 6. 4. 56-62.

Holb, I. J.-Heijne, B.-Jeger, M. J. (2003a): Summer epidemics of apple scab: the relationship between measurements and their implications for the development of predictive models and threshold levels under different disease control regimes. J. Phytopath, 151. 6. 335-343.

Holb, I. J.-Jong, de P. F.-Heijne, B. (2003b): Efficacy and phytotoxicity of lime sulphur in organic apple production. Annals of Applied Biology, 142. 2. 225-233.

Hrotkó, K.-Mukred, A.-Magyar, L.-Hanusz, B. (1996): Az alany és a tenyészterület hatása 'Idared' almafák fiatalkori növekedésére és termőre fordulására. Új Kertgazdaság, 1. 4. $1-8$.
Kozma, P.-Nyéki, J.-Soltész, M.-Szabó, Z. (2003): Floral biology, pollination and fertilisation in temperate zone fruit species and grape. Akadémiai Kiadó, Budapest, 621.

Nyéki, J.-Soltész, M. (1996): Floral biology of temperate zone fruit trees and small fruits. Akadémiai Kiadó, Budapest, 377.

Nyéki, J.-Soltész, M.-Szabó, Z. (2002): Fajtatársítás a gyümölcsültetvényekben. Mezőgazda Kiadó, Budapest, 382.

Nyéki, J.-Szabó, Z.-Racskó, J.-Soltész, M.-Gonda, I.-Farkas, E. (2004): Effect of M9, MM106 and seedling rootstocks on flowering and productivity of 33 apple cultivars. $8^{\text {th }}$ International Symposium on Integrating Canopy, Rootstock and Environmental Physiology in Orchard Systems, Budapest, 2004. June 13-18. Abstracts, 101.

Soltész, M. (1997): Integrált gyümölcstermesztés. Mezőgazda Kiadó, Budapest, 843.

Soltész, M. (1998): Gyümölcsfajta-ismeret és -használat. Mezőgazda Kiadó, Budapest, 513.

Szabó, Z.-Nyéki, J. (2003): Bud-, flower- and fruit density in stone fruits. Int. J. of Hort. Science, 3-4. 9. 59-69. 